\title{
Patient access schemes in Asia-pacific markets: current experience and future potential
}

\author{
Christine Y Lư ${ }^{1 *}$, Caitlin Lupton ${ }^{1}$, Shana Rakowsky², Zaheer-Ud-Din Babar ${ }^{3}$, Dennis Ross-Degnan ${ }^{1}$ \\ and Anita K Wagner ${ }^{1}$
}

\begin{abstract}
Objectives: Patient access (or risk-sharing) schemes are alternative market access agreements between healthcare payers and medical product manufacturers for conditional coverage of promising health technologies. This study aims to identify and characterize patient access schemes to date in the Asia-Pacific region.

Methods: We reviewed the literature on patient access schemes over the last two decades using publicly available databases, Internet, and grey literature searches. We extracted key features of each scheme identified, including the drug, clinical indication, stakeholders involved, and details of the scheme. We categorized schemes according to a previously published taxonomy of scheme types and by country.

Results: We identified 3 schemes in South Korea, 5 in New Zealand, and 98 in Australia. Most (97.2\%; n= 103) schemes focused on pharmaceuticals, few on medical technologies. More than half of the schemes related to treatments for cancer and inflammatory diseases such as rheumatoid arthritis. The majority $(77.4 \% ; n=82)$ involved pricing arrangements. Evidence generation schemes were rarely used. About half $(41.8 \% ; n=41)$ of schemes in Australia were hybrid by nature, consisting of pricing arrangements with a conditional treatment continuation component.

Conclusions: Australia has the most experience with patient access schemes and its experience may provide useful insights for other Asia-Pacific countries. The main targets are pharmaceuticals likely to have high budget impact (due to high per-patient costs and/or large volumes of use), and pharmaceuticals that may be adopted more widely than indicated. With the proliferation of high-cost medicines, the use of schemes may increase to address rising cost pressures, consumer demands, and uncertainties, while attempting to provide patient access to innovative care within finite budgets. Future research is warranted to evaluate the performance of patient access schemes.
\end{abstract}

Keywords: Patient access schemes, Risk sharing, Managed entry, Conditional coverage, Health technology assessment, High cost medicines, Access to medicines

\section{Introduction}

Many countries and insurance schemes are pursuing universal health coverage with the goal of ensuring that all people have access to needed health services without suffering financial hardship [1,2]. However, healthcare systems grapple with the challenges of funding clinically beneficial health services and medicines while ensuring that financing systems are sustainable.

\footnotetext{
* Correspondence: Christine_lu@harvardpilgrim.org

'Department of Population Medicine, Harvard Medical School and Harvard Pilgrim Health Care Institute, Boston, USA

Full list of author information is available at the end of the article
}

In an effort to improve the efficiency of rising healthcare expenditures, many systems evaluate whether expected additional health benefits of a new technology justify its additional cost compared to existing treatments (cost-effectiveness or 'value-for-money') [3-5]. Healthcare systems in the Asia-Pacific region that require proof of value-for-money in coverage of medical technologies include Australia, New Zealand, Thailand, South Korea, and Taiwan [6-10]. Even in systems that do not explicitly consider cost, there is often a focus on the magnitude of health benefits, which are informally weighed against cost. Traditionally, coverage decisions on medical technologies such as medicines and devices 
have been binary in nature; based on evidence available at launch and the price set by the manufacturer, payers decide whether or not to reimburse a product. In recent years, various types of conditional coverage decisions have emerged. Patient access schemes (also known as managed entry schemes or risk-sharing agreements) are alternative market access agreements, typically between payers and manufacturers, to enable provisional or conditional coverage of promising health technologies $[4,5,11]$. There are three broad categories of schemes [5]. First, outcome-based schemes (also known as performancebased or effectiveness guarantee schemes) in which the price, level, or nature of reimbursement are tied to clinical or intermediate endpoints measured in the future and ultimately related to patients' quality or quantity of life. Second, evidence generation schemes in which a positive coverage decision is conditioned upon the collection of additional evidence through clinical studies, which might result in continued, expanded, or withdrawn coverage. Third, financially-based schemes negotiate company contributions to the cost of a product (e.g., discounts or rebates, price-volume agreements, utilization caps) for a particular patient or population without linking reimbursement to health outcomes.

Interest in patient access schemes is growing due to increasing cost pressures, the need to balance the interests of patients, clinicians, manufacturers, and other stakeholders, and uncertainties due to incomplete information at the time that decisions must be made. The necessary evidence to demonstrate incremental effectiveness and cost-effectiveness of a new technology is often not available at the time of launch [12]. Therefore, uncertainties exist about whether the technology will deliver the promised health gains in routine clinical practice. Clinical and cost-effectiveness uncertainties result in budget uncertainties [12]. In addition, costs of innovative products may bankrupt households and threaten sustainability of systems. Patient access schemes offer an important option for systems to allow (some) patients (early) access to promising technologies that systems may not otherwise fund. At the same time, these schemes may reduce the financial risks that payers face in making decisions based on limited evidence, and in some cases, they facilitate collection of more evidence to support future decisions [4,5,11].

Little is known about experiences with patient access schemes in Asia-Pacific countries. The purpose of this study was to identify and categorize patient access schemes in this region. The results of this study will enhance understanding of the roles of patient access schemes in emerging Asia-Pacific markets and inform policy developments for enabling patient access to promising technologies.

\section{Methods}

We reviewed the literature on patient access schemes. We searched in PubMed and Google Scholar for English-language articles published through July 2012 using the following terms; 'conditional coverage', 'conditional reimbursement', 'managed entry', 'risk sharing', 'risk sharing agreement', 'risk sharing, pharmaceuticals', 'risk sharing scheme', 'coverage with evidence', 'valuebased pricing, pharmaceuticals', 'patient access scheme', 'pay back schemes', 'performance-based, pharmaceuticals', 'outcome-based reimbursement, pharmaceuticals', 'reimbursement mechanisms, pharmaceuticals', and 'outcome guarantee'. We scanned titles and abstracts to select relevant articles to review. The reference lists of relevant articles were also reviewed for studies fitting our criteria that our search strategy may have missed. We also added relevant unpublished or 'grey literature' (reports, conference presentations, payer websites) that were identified during our search.

We extracted key features of the schemes: the technology, disease area, country, payer, manufacturer, scheme type, and agreement details (when reported). We categorized the schemes according to a published taxonomy [5] of scheme types: (1) outcome-based schemes, (2) evidence generation schemes, and (3) financially-based schemes. These scheme types have generally been termed "risk sharing schemes" or "patient access schemes" by payers or pharmaceutical companies. Consistent with the literature [13], we classified schemes with a "conditional treatment continuation" component that limits continued subsidy of medicines to patients who demonstrate a pre-specified adequate clinical response as outcome-based schemes. We deliberately included financially-based schemes, in contrast to previous reviews of patient access schemes that focused on outcome-based schemes only [13]. Although financially-based schemes do not directly address uncertainties in clinical outcomes, they can address uncertainties in cost-effectiveness and/or budget impact estimates. Their use is increasing rapidly because they may be more feasible than outcome-based schemes that require more information and are administratively burdensome. We did not limit our search to any country. We report on schemes identified in Asia-Pacific markets.

\section{Results}

\section{Search results}

Using the search terms in PubMed and Google Scholar, we found 2229 articles published between 1998 and July 2012. Reviewing article titles and abstracts, we excluded commentaries and articles that did not describe specific patient access schemes. Next, we reviewed the references of remaining articles and grey literature sources for additional examples of patient access schemes. Our search identified 299 schemes described in 146 publicly available 
sources (92 scientific articles, 18 electronic articles, 14 reports, 9 websites, and 13 conference presentations). From these we selected the 106 examples from the Asia-Pacific region.

\section{Participating countries}

We identified 106 patient access schemes in 3 countries from the Asia-Pacific region (Table 1). Public payers were involved in all schemes. Most (92.5\%; $\mathrm{n}=98$ ) have taken place in Australia (Table 2) [14-21]. The Australian Pharmaceutical Benefits Advisory Committee (PBAC) is an expert committee that reviews incremental effectiveness and cost-effectiveness of medicines for coverage under the Pharmaceutical Benefits Scheme. The PBAC has formally assessed evidence of cost-effectiveness since 1993 [4]. The Medical Benefits Advisory Committee is an equivalent committee that evaluates medical devices for coverage under the Medical Benefits Scheme.

Apart from Australia, we found 5 schemes in New Zealand and 3 in South Korea. Table 3 summarizes the schemes [22-25]. These countries also have established a formal process for health technology assessment. Similar to Australia, New Zealand has a national health insurance for its people. The pharmaceutical management agency was established in 1993 to make decisions about drug coverage. The agency considers a number of factors, including the clinical need, clinical benefits and risks, cost-effectiveness, and budget impact of new drugs [26]. South Korea is the first country in Asia to officially adopt economic evaluation for making drug reimbursement

Table 1 Patient access schemes in the Asia-Pacific region by country, type and condition

\begin{tabular}{lllll}
\hline & Australia & $\begin{array}{l}\text { South } \\
\text { Korea }\end{array}$ & $\begin{array}{l}\text { New } \\
\text { Zealand }\end{array}$ & Total \\
\hline Types & 21 & - & - & 21 \\
Outcome-based & 3 & - & - & 3 \\
Evidence generation & 33 & 3 & 5 & 41 \\
Financially-based & 41 & - & - & 41 \\
Hybrid* & & & & \\
Conditions & 29 & - & 2 & 31 \\
Cancer & 28 & - & 1 & 29 \\
Inflammatory Conditions & 28 & - & - & 7 \\
Infectious Disease & 7 & 1 & 1 & 9 \\
$\quad \begin{array}{l}\text { Pulmonary Hypertension } \\
\text { Other }\end{array}$ & 7 & 2 & 1 & 30 \\
Technology & 27 & & & 103 \\
$\quad$ Pharmaceuticals & 95 & 3 & 5 & 3 \\
Medical devices & 3 & - & - & 106 \\
\hline Subtotal & 98 & 3 & 5 &
\end{tabular}

*Hybrid schemes involved both pricing arrangements and conditional treatment continuation decisions since January 2008. The Health Insurance Review Agency is responsible for reviewing the cost-effectiveness and budget impacts of new drugs for reimbursement under South Korea's National Health Insurance [27].

\section{Types of medical products}

Almost all patient access schemes focused on pharmaceuticals $(97.2 \% ; n=103)$, few on medical technologies. More than half of schemes covered treatments for noncommunicable diseases, predominantly cancer $(n=31)$ and inflammatory diseases $(n=29)$ such as rheumatoid arthritis. Schemes in Australia were largely established for technologies with high budget impact due to high cost per patient (e.g., adalimumab, which costs approximately $\$ 20,000$ per patient-year) or large volumes of use (e.g., dabigatran for prevention of stroke or systemic embolism), as well as for products that may be used beyond their approved indications (e.g., entecavir that is subsidized only for chronic hepatitis B in adults with evidence of active liver inflammation). Similarly, New Zealand targeted new products with high cost per-patient or large volumes of use. Instead of individual drugs, the schemes in South Korea targeted the entire class of older medications with high budget impact due to large volumes of use (e.g., hyperlipidemia medications).

\section{Outcome-based schemes}

Nearly two-thirds of schemes in Australia (63.3\% n =62; Table 2) included a "conditional treatment continuation" component that limits continued subsidy of high-cost medicines to patients who demonstrate an adequate clinical response. These schemes specify strict criteria for both initial and continued access. For example, the scheme for adalimumab for rheumatoid arthritis requires confirmation of clinical improvement at 3 months (measured by reductions in the total number of affected joints and in levels of inflammation markers) for continued subsidy [28]. In the scheme for bosenten in Australia, price was directly linked to the observed survival of patients treated with bosentan to confirm the clinical benefits (i.e., survival) and cost-effectiveness assumed at the time of assessment [29].

\section{Evidence generation schemes}

We found three schemes with an evidence generation component in Australia, all focused on medical technologies: positron emission tomography, deep brain stimulators, and endovascular aneurysm repair (Table 2). In all three cases, the Australian government agreed to provide interim funding for access to the technology and to support collection of relevant clinical (and cost) information [15]. The manufacturers did not share the financial costs to generate evidence. 
Table 2 Identified patient access schemes in Australia

\begin{tabular}{|c|c|c|c|c|c|}
\hline Technology & Indication & Company & Payer & Type & Source \\
\hline \multicolumn{6}{|c|}{ Cancer $(n=29)$} \\
\hline Dasatinib & CML & $\begin{array}{l}\text { Bristol-Myers Squibb Australia } \\
\text { Pty Ltd }\end{array}$ & PBS & Outcome-based & Medicare Australia [14] \\
\hline Nilotinib & CML & $\begin{array}{l}\text { Novartis Pharmaceuticals Australia } \\
\text { Pty Ltd }\end{array}$ & PBS & Outcome-based & Medicare Australia [14] \\
\hline Imatinib & GIST, adjuvant & $\begin{array}{l}\text { Novartis Pharmaceuticals Australia } \\
\text { Pty Ltd }\end{array}$ & PBS & Outcome-based & Medicare Australia [14] \\
\hline Imatinib & GIST, metastatic & $\begin{array}{l}\text { Novartis Pharmaceuticals Australia } \\
\text { Pty Ltd }\end{array}$ & PBS & Outcome-based & Medicare Australia [14] \\
\hline Lapatinib & Late stage metastatic breast cancer & GlaxoSmithKline Australia Pty Ltd & PBS & Outcome-based & Medicare Australia [14] \\
\hline Imatinib & $\begin{array}{l}\text { Rare cancers (eg dermatofibrosarcoma protuberans, } \\
\text { chronic eosinophilic leukemia) }\end{array}$ & $\begin{array}{l}\text { Novartis Pharmaceuticals Australia } \\
\text { Pty Ltd }\end{array}$ & PBS & Outcome-based & Medicare Australia [14] \\
\hline Pazopanib & RCC & GlaxoSmithKline Australia Pty Ltd & PBS & $\begin{array}{l}\text { Financially-based; } \\
\text { Outcome-based }\end{array}$ & Public Summary Document [18] \\
\hline Bevacizumab & Metastatic colorectal cancerr & Roche Products Pty Ltd & PBS & $\begin{array}{l}\text { Financially-based; } \\
\text { Outcome-based }\end{array}$ & Public Summary Document [18] \\
\hline Sorafenib & Advanced hepatocellular carcinoma & Bayer Australia Ltd & PBS & $\begin{array}{l}\text { Financially-based; } \\
\text { Outcome-based }\end{array}$ & Public Summary Document [18] \\
\hline PET & $\begin{array}{l}\text { Staging of newly diagnosed NSCLC, esophageal cancer, } \\
\text { cancer of gastro-esophageal junction, head and neck } \\
\text { cancer, suspected residual/metastatic/recurrent colorectal } \\
\text { cancer, melanoma, head and neck cancer, ovarian cancer }\end{array}$ & Not specified & MBS & Evidence generation & Stafinski et al. 2010 [15] \\
\hline Exemestane & $\begin{array}{l}\text { Oestrogen-receptor positive advanced breast cancer in } \\
\text { post-menopausal women }\end{array}$ & Pfizer Australia Pty Ltd & PBS & Financially-based & Public Summary Document [18] \\
\hline Imiquimod & Superficial basal cell carcinoma & 3 M Pharmaceuticals Pty Ltd & PBS & Financially-based & Public Summary Document [18] \\
\hline Letrozole & Advanced breast cancer in postmenopausal women & $\begin{array}{l}\text { Novartis Pharmaceuticals Australia } \\
\text { Pty Ltd }\end{array}$ & PBS & Financially-based & Public Summary Document [18] \\
\hline Docetaxel & $\mathrm{SCCHN}$ & Sanofi-Aventis Australia Pty Ltd & PBS & Financially-based & Public Summary Document [18] \\
\hline Sunitinib & RCC & Pfizer Australia Pty Ltd & PBS & Financially-based & $\begin{array}{l}\text { PBS Schedule [16], Public Summary } \\
\text { Document [18] }\end{array}$ \\
\hline Cetuximab & K-RAS wild mCRC & Merck Serono Australia Pty Ltd & PBS & Financially-based & Public Summary Document [18] \\
\hline Vemurafenib & Untreated unresectable stage IIIC or stage IV melanoma & Roche Products Pty Ltd & PBS & Financially-based & Public Summary Document [18] \\
\hline Imatinib & ALL & $\begin{array}{l}\text { Novartis Pharmaceuticals } \\
\text { Australia Pty Ltd }\end{array}$ & PBS & $\begin{array}{l}\text { Financially-based; } \\
\text { Outcome-based }\end{array}$ & $\begin{array}{l}\text { PBPA Relativity Sheets [17], Public } \\
\text { Summary Document [18], Medicare } \\
\text { Australia [14] }\end{array}$ \\
\hline Dasatinib & ALL & $\begin{array}{l}\text { Bristol-Myers Squibb Australia } \\
\text { Pty Ltd }\end{array}$ & PBS & $\begin{array}{l}\text { Financially-based; } \\
\text { Outcome-based }\end{array}$ & $\begin{array}{l}\text { PBPA Relativity Sheets [17], Public } \\
\text { Summary Document [18], Medicare } \\
\text { Australia [14] }\end{array}$ \\
\hline Azacitidine & CML & Celgene Pty Ltd & PBS & & PBS Schedule [16], Medicare Australia [14] \\
\hline
\end{tabular}


Table 2 Identified patient access schemes in Australia (Continued)

\begin{tabular}{|c|c|c|c|c|c|}
\hline \multirow[b]{2}{*}{ Azacitidine } & \multirow[b]{2}{*}{ AML } & \multirow[b]{2}{*}{ Celgene Pty Ltd } & \multirow[b]{2}{*}{ PBS } & \\
\hline & & & & $\begin{array}{l}\text { Financially-based; } \\
\text { Outcome-based }\end{array}$ & PBS Schedule [16], Medicare Australia [14] \\
\hline Azacitidine & Myelodysplastic syndrome & Celgene Pty Ltd & PBS & $\begin{array}{l}\text { Financially-based; } \\
\text { Outcome-based }\end{array}$ & PBS Schedule [16], Medicare Australia [14] \\
\hline Imatinib & CML & $\begin{array}{l}\text { Novartis Pharmaceuticals Australia } \\
\text { Pty Ltd }\end{array}$ & PBS & $\begin{array}{l}\text { Financially-based; } \\
\text { Outcome-based }\end{array}$ & $\begin{array}{l}\text { PBPA Relativity Sheets [17], Medicare } \\
\text { Australia [14] }\end{array}$ \\
\hline Trastuzumab & HER2+ early breast cancer & Roche Products Pty Ltd & PBS & $\begin{array}{l}\text { Financially-based; } \\
\text { Outcome-based }\end{array}$ & $\begin{array}{l}\text { PBPA Relativity Sheets [17], Medicare } \\
\text { Australia [14] }\end{array}$ \\
\hline Sunitinib & GIST & Pfizer Australia Pty Ltd & PBS & $\begin{array}{l}\text { Financially-based; } \\
\text { Outcome-based }\end{array}$ & PBS Schedule [16], Medicare Australia [14] \\
\hline Lenalidomide & Myelodysplastic syndrome & Celgene Pty Ltd & PBS & $\begin{array}{l}\text { Financially-based; } \\
\text { Outcome-based }\end{array}$ & PBS Schedule [16], Medicare Australia [14] \\
\hline Lenalidomide & Multiple myeloma & Celgene Pty Ltd & PBS & $\begin{array}{l}\text { Financially-based; } \\
\text { Outcome-based }\end{array}$ & PBS Schedule [16], Medicare Australia [14] \\
\hline Bortezomib & Multiple myeloma & Janssen-Cilag Pty Ltd & PBS & $\begin{array}{l}\text { Financially-based, } \\
\text { Outcome-based }\end{array}$ & $\begin{array}{l}\text { PBS Schedule [16], PBPA Relativity Sheets } \\
\text { [17], Public Summary Document [18], } \\
\text { Medicare Australia [14] }\end{array}$ \\
\hline Erlotinib & Non-small cell lung cancer & Roche Products Pty Ltd & PBS & $\begin{array}{l}\text { Financially-based; } \\
\text { Outcome-based }\end{array}$ & PBS Schedule [16], Medicare Australia [14] \\
\hline \multicolumn{6}{|c|}{ Inflammatory Conditions $(n=28)$} \\
\hline Adalimumab & Ankylosing spondylitis & AbbVie Pty Ltd & PBS & Outcome-based & Medicare Australia [14] \\
\hline Etanercept & Ankylosing spondylitis & Pfizer Australia Pty Ltd & PBS & Outcome-based & Medicare Australia [14] \\
\hline Golimumab & Ankylosing spondylitis & Janssen-Cilag Pty Ltd & PBS & Outcome-based & Medicare Australia [14] \\
\hline Infliximab & Ankylosing spondylitis & Janssen-Cilag Pty Ltd & PBS & Outcome-based & Medicare Australia [14] \\
\hline Adalimumab & Crohn's disease & AbbVie Pty Ltd & PBS & Outcome-based & Medicare Australia [14] \\
\hline Infliximab & Complex refractory fistulising Crohn's disease & Janssen-Cilag Pty Ltd & PBS & Outcome-based & Medicare Australia [14] \\
\hline Adalimumab & Juvenile arthritis & AbbVie Pty Ltd & PBS & Outcome-based & Medicare Australia [14] \\
\hline Etanercept & Juvenile arthritis & Pfizer Australia Pty Ltd & PBS & Outcome-based & Medicare Australia [14] \\
\hline Adalimumab & Psoriatic arthritis & AbbVie Pty Ltd & PBS & Outcome-based & Medicare Australia [14] \\
\hline Golimumab & Psoriatic arthritis & Janssen-Cilag Pty Ltd( & PBS & Outcome-based & Medicare Australia [14] \\
\hline Infliximab & Psoriatic arthritis & Janssen-Cilag Pty Ltd & PBS & Outcome-based & Medicare Australia [14] \\
\hline Pimecrolimus & Atopic dermatitis who are over 18 years of age & Novartis Pharmaceuticals Australia & PBS & Financially-based & Public Summary Document [18] \\
\hline Infliximab & Crohn's disease & Janssen-Cilag Pty Ltd & PBS & $\begin{array}{l}\text { Financially-based; } \\
\text { Outcome-based }\end{array}$ & $\begin{array}{l}\text { PBPA Relativity Sheets [17], Medicare } \\
\text { Australia [14] }\end{array}$ \\
\hline Adalimumab & Complex refractory fistulising Crohn's disease & AbbVie Pty Ltd & PBS & $\begin{array}{l}\text { Financially-based; } \\
\text { Outcome-based }\end{array}$ & $\begin{array}{l}\text { PBPA Relativity Sheets [17], Medicare } \\
\text { Australia [14] }\end{array}$ \\
\hline
\end{tabular}


Table 2 Identified patient access schemes in Australia (Continued)

\begin{tabular}{|c|c|c|c|c|c|}
\hline Tocilizumab & Juvenile arthritis & Roche Products Pty Ltd & PBS & $\begin{array}{l}\text { Financially-based; } \\
\text { Outcome-based }\end{array}$ & $\begin{array}{l}\text { PBPA Relativity Sheets [17], Medicare } \\
\text { Australia [14] }\end{array}$ \\
\hline Abatacept & RA & $\begin{array}{l}\text { Novartis Pharmaceuticals Australia } \\
\text { Pty Ltd }\end{array}$ & PBS & $\begin{array}{l}\text { Financially-based; } \\
\text { Outcome-based }\end{array}$ & PBS Schedule [16], Medicare Australia [14] \\
\hline Infliximab & RA & Janssen-Cilag Pty Ltd & PBS & $\begin{array}{l}\text { Financially-based; } \\
\text { Outcome-based }\end{array}$ & $\begin{array}{l}\text { PBS Schedule [16], Lu et al. } 2007 \text { [33], } \\
\text { Pugatch et al. } 2010 \text { [21], Medicare } \\
\text { Australia [14] }\end{array}$ \\
\hline Rituximab & RA & Roche Products Pty Ltd & PBS & $\begin{array}{l}\text { Financially-based; } \\
\text { Outcome-based }\end{array}$ & $\begin{array}{l}\text { PBS Schedule [16], Lu et al. } 2007 \text { [33], } \\
\text { Pugatch et al. } 2010 \text { [21], Medicare } \\
\text { Australia [14] }\end{array}$ \\
\hline Tocilizumab & RA & Roche Products Pty Ltd & PBS & $\begin{array}{l}\text { Financially-based; } \\
\text { Outcome-based }\end{array}$ & PBS Schedule [16], Medicare Australia [14] \\
\hline Certolizumab Pegol & RA & UCB Australia Proprietary Ltd & PBS & $\begin{array}{l}\text { Financially-based; } \\
\text { Outcome-based }\end{array}$ & PBS Schedule [16], Medicare Australia [14] \\
\hline Adalimumab & RA & AbbVie Pty Ltd & PBS & $\begin{array}{l}\text { Financially-based; } \\
\text { Outcome-based }\end{array}$ & $\begin{array}{l}\text { PBS Schedule [16], Lu et al. } 2007 \text { [33], } \\
\text { Pugatch et al. } 2010 \text { [21], Medicare } \\
\text { Australia [14] }\end{array}$ \\
\hline Golimumab & RA & Janssen-Cilag Pty Ltd & PBS & $\begin{array}{l}\text { Financially-based; } \\
\text { Outcome-based }\end{array}$ & PBS Schedule [16], Medicare Australia [14] \\
\hline Etanercept & RA & Pfizer Australia Pty Ltd & PBS & $\begin{array}{l}\text { Financially-based; } \\
\text { Outcome-based }\end{array}$ & $\begin{array}{l}\text { PBS Schedule [16], Lu et al. } 2007 \text { [33], } \\
\text { Pugatch et al. } 2010 \text { [21], Medicare } \\
\text { Australia [14] }\end{array}$ \\
\hline Adalimumab & Severe chronic plaque psoriasis & AbbVie Pty Ltd & PBS & $\begin{array}{l}\text { Financially-based; } \\
\text { Outcome-based }\end{array}$ & $\begin{array}{l}\text { PBS Schedule [16], Lu et al. } 2007 \text { [33], } \\
\text { Pugatch et al. } 2010 \text { [21], Medicare } \\
\text { Australia [14] }\end{array}$ \\
\hline Etanercept & Severe chronic plaque psoriasis & Pfizer Australia Pty Ltd & PBS & $\begin{array}{l}\text { Financially-based; } \\
\text { Outcome-based }\end{array}$ & PBS Schedule [16], Medicare Australia [14] \\
\hline Infliximab & Severe chronic plaque psoriasis & Janssen-Cilag Pty Ltd & PBS & $\begin{array}{l}\text { Financially-based; } \\
\text { Outcome-based }\end{array}$ & PBS Schedule [16], Medicare Australia [14] \\
\hline Ustekinumab & Severe chronic plaque psoriasis & Janssen-Cilag Pty Ltd & PBS & $\begin{array}{l}\text { Financially-based; } \\
\text { Outcome-based }\end{array}$ & PBS Schedule [16], Medicare Australia [14] \\
\hline Etanercept & Severe chronic plaque psoriasis under 18 & Pfizer Australia Pty Ltd & PBS & $\begin{array}{l}\text { Financially-based; } \\
\text { Outcome-based }\end{array}$ & PBS Schedule [16], Medicare Australia [14] \\
\hline \multicolumn{6}{|c|}{ Infectious Diseases $(n=7)$} \\
\hline Abacavir & HIV infection & ViiV Healthcare Pty Ltd & PBS & Financially-based & $\begin{array}{l}\text { Robertson et al. } 2009 \text { [19], Adamski et al. } \\
2010 \text { [20] }\end{array}$ \\
\hline Tipranavir & HIV infection & Boehringer Ingelheim Pty Ltd & PBS & Financially-based & $\begin{array}{l}\text { PBS Schedule [16], Public Summary } \\
\text { Document [18] }\end{array}$ \\
\hline Entecavir & $\begin{array}{l}\text { Chronic hepatitis B in adults } 16 \text { years and older with } \\
\text { evidence of active liver inflammation }\end{array}$ & $\begin{array}{l}\text { Bristol-Myers Squibb Australia } \\
\text { Pty Ltd }\end{array}$ & PBS & Financially-based & Public Summary Document [18] \\
\hline
\end{tabular}


Table 2 Identified patient access schemes in Australia (Continued)

\begin{tabular}{|c|c|c|c|c|c|}
\hline Boceprevir & Chronic genotype 1 hepatitis $C$ infection & $\begin{array}{l}\text { Merck Sharp \& Dohme (Australia) } \\
\text { Pty Ltd }\end{array}$ & PBS & Financially-based & Public Summary Document [18] \\
\hline Telaprevir & Chronic genotype 1 hepatitis C & Janssen-Cilag Pty Ltd & PBS & Financially-based & Public Summary Document [18] \\
\hline Posaconazole & $\begin{array}{l}\text { Invasive fungal infections, not responsive to or intolerant } \\
\text { of, alternative therapy }\end{array}$ & Schering-Plough Pty Ltd & PBS & Financially-based & Public Summary Document [18] \\
\hline Posaconazole & $\begin{array}{l}\text { Prophylaxis of invasive fungal infections among high risk } \\
\text { patients }\end{array}$ & Schering-Plough Pty Ltd & PBS & Financially-based & Public Summary Document [18] \\
\hline \multicolumn{6}{|c|}{ Pulmonary Hypertension $(n=7)$} \\
\hline Sildenafil & $\mathrm{PPH}$ or $\mathrm{PAH}$ & Pfizer Australia Pty Ltd & PBS & Outcome-based & PBS Schedule [16], Medicare Australia [14] \\
\hline Tadalafil & $\mathrm{PPH}$ or PAH & Eli Lilly Australia Pty Ltd & PBS & Outcome-based & PBS Schedule [16], Medicare Australia [14] \\
\hline Treprostinil Sodium & PPH or PAH & Orphan Australia Pty Ltd & PBS & Financially-based & Public Summary Document [18] \\
\hline Ambrisentan & PPH or PAH & GlaxoSmithKline Australia Pty Ltd & PBS & $\begin{array}{l}\text { Financially-based; } \\
\text { Outcome-based }\end{array}$ & PBS Schedule [16], Medicare Australia [14] \\
\hline Bosentan & PPH or PAH & $\begin{array}{l}\text { Actelion Pharmaceuticals Australia } \\
\text { Pty Ltd }\end{array}$ & PBS & $\begin{array}{l}\text { Financially-based; } \\
\text { Outcome-based }\end{array}$ & $\begin{array}{l}\text { PBS Schedule [16], PBPA Relativity Sheets } \\
\text { [17], Public Summary Document [18], } \\
\text { Medicare Australia [14] }\end{array}$ \\
\hline Epoprostenol & $\mathrm{PPH}$ or PAH & GlaxoSmithKline Australia Pty Ltd & PBS & $\begin{array}{l}\text { Financially-based; } \\
\text { Outcome-based }\end{array}$ & $\begin{array}{l}\text { PBS Schedule [16], PBPA Relativity Sheets } \\
\text { [17], Public Summary Document [18], } \\
\text { Medicare Australia [14] }\end{array}$ \\
\hline Iloprost trometamol & $\mathrm{PPH}$ or PAH & Bayer Australia Ltd & PBS & $\begin{array}{l}\text { Financially-based; } \\
\text { Outcome-based }\end{array}$ & PBS Schedule [16], Medicare Australia [14] \\
\hline \multicolumn{6}{|l|}{ Other $(n=27)$} \\
\hline Modafinil & Narcolepsy & bioCSL (Australia) Pty Ltd & PBS & Outcome-based & Medicare Australia [14] \\
\hline Verteporfin & Age-related macular degeneration & $\begin{array}{l}\text { Novartis Pharmaceuticals Australia } \\
\text { Pty Ltd }\end{array}$ & PBS & Outcome-based & Medicare Australia [14] \\
\hline Deep brain stimulation & $\begin{array}{l}\text { Patients with Parkinson's disease no longer responsive to } \\
\text { drug therapy }\end{array}$ & Not specified & MBS & Evidence generation & Stafinski et al. 2010 [15] \\
\hline EVAR & Abdominal aortic aneurysm & Not specified & MBS & Evidence generation & Stafinski et al. 2010 [15] \\
\hline Dabigatran & $\begin{array}{l}\text { Prevention of stroke or systemic embolism in patients with } \\
\text { non-valvular atrial fibrillation }\end{array}$ & Boehringer Ingelheim Pty Ltd & PBS & Financially-based & Public Summary Document [18] \\
\hline $\begin{array}{l}\text { Atomoxetine } \\
\text { Hydrochloride }\end{array}$ & ADHD diagnosed between the ages of 6 and 18 years & Eli Lilly Australia Pty Ltd & PBS & Financially-based & Public Summary Document [18] \\
\hline Fingolimod & RRMS & $\begin{array}{l}\text { Novartis Pharmaceuticals Australia } \\
\text { Pty Ltd }\end{array}$ & PBS & $\begin{array}{l}\text { Financially-based; } \\
\text { Outcome-based }\end{array}$ & Public Summary Document [18] \\
\hline Cinacalcet Hydrochloride & $\begin{array}{l}\text { End stage renal disease receiving dialysis who have } \\
\text { uncontrolled secondary hyperparathyroidism }\end{array}$ & Amgen Australia Pty Ltd & PBS & Financially-based & $\begin{array}{l}\text { PBS Schedule [16], Public Summary } \\
\text { Document [18] }\end{array}$ \\
\hline Clostridium botulinum & Severe spasticity of the upper limb in adults following a & Ipsen Pty Ltd & PBS & Financially-based & Public Summary Document [18] \\
\hline
\end{tabular}

type A toxinstroke, as an adjunct to physical therapy

Public Summary Document [18]

haemagglutinin

complex 
Table 2 Identified patient access schemes in Australia (Continued)

\begin{tabular}{|c|c|c|c|c|c|}
\hline Fentanyl citrate & Fentanyl lozenges for the treatment of breakthrough pain & Orphan Australia Pty Ltd & PBS & Financially-based & Public Summary Document [18] \\
\hline Natalizumab & RRMS & Biogen Idec Australia Pty Ltd & PBS & Financially-based & Public Summary Document [18] \\
\hline Paliperidone & Schizophrenia & Janssen-Cilag Pty Ltd & PBS & Financially-based & Public Summary Document [18] \\
\hline $\begin{array}{l}\text { Botulinum toxin type a } \\
\text { purified neurotoxin } \\
\text { complex }\end{array}$ & $\begin{array}{l}\text { Moderate to severe spasticity of the upper limb in adults } \\
\text { following a stroke as an adjunct to physical therapy }\end{array}$ & Allergan Australia Pty Ltd & PBS & Financially-based & Public Summary Document [18] \\
\hline $\begin{array}{l}\text { Tenofovir Disoproxil } \\
\text { Fumarate }\end{array}$ & Treatment of chronic hepatitis B & Gilead Sciences Pty Ltd & PBS & Financially-based & Public Summary Document [18] \\
\hline $\begin{array}{l}\text { Pramipexole } \\
\text { hydrochloride }\end{array}$ & Idiopathic Parkinson disease & Boehringer Ingelheim Pty Ltd & PBS & Financially-based & Public Summary Document [18] \\
\hline Tobramycin & $\begin{array}{l}\text { Pseudomonas aeruginosa respiratory infection in a patient } \\
\text { with Cystic Fibrosis }\end{array}$ & $\begin{array}{l}\text { Novartis Pharmaceuticals Australia } \\
\text { Pty Ltd }\end{array}$ & PBS & Financially-based & Public Summary Document [18] \\
\hline Ticagrelor & ACS, MI, or unstable angina & AstraZeneca Pty Ltd & PBS & Financially-based & Public Summary Document [18] \\
\hline Pregabalin & Neuropathic pain & Pfizer Australia Pty Ltd & PBS & Financially-based & Public Summary Document [18] \\
\hline Rivaroxaban & Acute symptomatic DVT & Bayer Australia Ltd & PBS & Financially-based & Public Summary Document [18] \\
\hline Aztreonam & $\begin{array}{l}\text { Pseudomonas aeruginosa infection in patients with cystic } \\
\text { fibrosis }\end{array}$ & Gilead Sciences Pty Ltd & PBS & Financially-based & Public Summary Document [18] \\
\hline $\begin{array}{l}\text { Crinone (progesterone } \\
\text { gel) }\end{array}$ & $\begin{array}{l}\text { Supplement progesterone in women who have luteal } \\
\text { phase defect }\end{array}$ & Merck Serono & PBS & Financially-based & $\begin{array}{l}\text { Robertson et al. } 2009 \text { [19], Adamski et al. } \\
2010 \text { [20] }\end{array}$ \\
\hline Deferasirox (Exjade) & $\begin{array}{l}\text { Chronic iron overload in patients with disorders of } \\
\text { erythropoiesis }\end{array}$ & Novartis & PBS & Financially-based & $\begin{array}{l}\text { Robertson et al. } 2009 \text { [19], Adamski et al. } \\
2010 \text { [20] }\end{array}$ \\
\hline Omalizumab & Uncontrolled severe allergic asthma & $\begin{array}{l}\text { Novartis Pharmaceuticals Australia } \\
\text { Pty Ltd }\end{array}$ & PBS & $\begin{array}{l}\text { Financially-based; } \\
\text { Outcome-based }\end{array}$ & PBS Schedule [16], Medicare Australia [14] \\
\hline Aflibercept & Age-related macular degeneration & Bayer Australia Ltd & PBS & $\begin{array}{l}\text { Financially-based; } \\
\text { Outcome-based }\end{array}$ & PBS Schedule [16], Medicare Australia [14] \\
\hline Ranibizumab & Age-related macular degeneration & $\begin{array}{l}\text { Novartis Pharmaceuticals Australia } \\
\text { Pty Ltd }\end{array}$ & PBS & $\begin{array}{l}\text { Financially-based; } \\
\text { Outcome-based }\end{array}$ & $\begin{array}{l}\text { PBS Schedule [16], PBPA Relativity Sheets } \\
\text { [17], Public Summary Document [18], } \\
\text { Medicare Australia [14] }\end{array}$ \\
\hline Eltrombopag & $\begin{array}{l}\text { Severe chronic immune idiopathic thrombocytopenic } \\
\text { purpura }\end{array}$ & GlaxoSmithKline Australia Pty Ltd & PBS & $\begin{array}{l}\text { Financially-based; } \\
\text { Outcome-based }\end{array}$ & $\begin{array}{l}\text { PBPA Relativity Sheets [17], Medicare } \\
\text { Australia [14] }\end{array}$ \\
\hline Romiplostim & $\begin{array}{l}\text { Severe chronic immune idiopathic thrombocytopenic } \\
\text { purpura }\end{array}$ & Amgen Australia Pty Ltd & PBS & $\begin{array}{l}\text { Financially-based; } \\
\text { Outcome-based }\end{array}$ & PBS Schedule [16], Medicare Australia [14] \\
\hline
\end{tabular}

ACS = Acute Coronary Syndromes; ALL = Acute Lymphoblastic Leukemia; AML = Acute Myeloid Leukemia; $C \mathrm{ML}=$ Chronic Myeloid Leukemia; DVT = Deep Vein Thrombosis; EVAR = endovascular aneurysm repair; GIST = Gastrointestinal Stromal Tumor; MBS = Medicare Benefits Schedule; $m C R C=$ Metastatic Colorectal Cancer; MI = Myocardial Infarction; PAH = Pulmonary Arterial Hypertension; PBS = Pharmaceutical Benefits Scheme; PPH = Primary Pulmonary Hypertension; RA = Rheumatoid Arthritis; RCC = Renal Cell Carcinoma; RRMS = Relapsing-Remitting Multiple Sclerosis; SCCHN = Squamous Cell Carcinoma of the Head and Neck. 
Table 3 Identified patient access schemes in the Asian-Pacific region (excluding Australian examples)

\begin{tabular}{|c|c|c|c|c|c|c|c|}
\hline Country & Drug & Indication & Company & Payer & Type & Details & Source \\
\hline \multirow[t]{3}{*}{$\begin{array}{l}\text { South } \\
\text { Korea }\end{array}$} & $\begin{array}{l}\text { Migraine } \\
\text { medications }\end{array}$ & $\begin{array}{l}\text { Migraine } \\
\text { headaches }\end{array}$ & $\begin{array}{l}\text { Not } \\
\text { specified }\end{array}$ & $\begin{array}{l}\text { National } \\
\text { health } \\
\text { insurance }\end{array}$ & $\begin{array}{l}\text { Financially- } \\
\text { based }\end{array}$ & Pharmaceutical companies maintained their PLS status via voluntary price cuts & Lee et al. 2012 [22] \\
\hline & $\begin{array}{l}\text { Hypertension } \\
\text { medications }\end{array}$ & Hypertension & $\begin{array}{l}\text { Not } \\
\text { specified }\end{array}$ & $\begin{array}{l}\text { National } \\
\text { health } \\
\text { insurance }\end{array}$ & $\begin{array}{l}\text { Financially- } \\
\text { based }\end{array}$ & $\begin{array}{l}285 \text { of the } 1226 \text { hypertension drugs instituted price reductions following } \\
\text { reassessment of clinical usefulness \& price. Drugs were delisted if they failed to } \\
\text { show a level of clinical usefulness or if their prices were higher than } 80 \text { percentile of } \\
\text { the highest price among drugs containing the same ingredients. If the company } \\
\text { accepted price cuts, the price was to be lowered to the level of the } 80 \text { percentile } \\
\text { within } 3 \text { years }\end{array}$ & Lee et al. 2012 [22] \\
\hline & $\begin{array}{l}\text { Hyperlipidemia } \\
\text { medications - } \\
\text { therapeutic class }\end{array}$ & Hyperlipidemia & $\begin{array}{l}\text { Not } \\
\text { specified }\end{array}$ & $\begin{array}{l}\text { National } \\
\text { health } \\
\text { insurance }\end{array}$ & $\begin{array}{l}\text { Financially- } \\
\text { based }\end{array}$ & Pharmaceutical companies maintained their PLS status via voluntary price cuts & Lee et al. 2012 [22] \\
\hline \multirow[t]{5}{*}{$\begin{array}{l}\text { New } \\
\text { Zealand }\end{array}$} & $\begin{array}{l}\text { Beta-interferon } \\
\text { products, } \\
\text { glatiramer acetate }\end{array}$ & $\begin{array}{l}\text { Multiple } \\
\text { sclerosis }\end{array}$ & Bayer & PHARMAC & $\begin{array}{l}\text { Financially- } \\
\text { based }\end{array}$ & Extended coverage for a specified number of patients & Raftery 2008 [23] \\
\hline & Trastuzumab & Breast cancer & Roche & PHARMAC & $\begin{array}{l}\text { Financially- } \\
\text { based }\end{array}$ & Established separate hospital program to fund distribution of cancer drugs & Raftery 2008 [23] \\
\hline & Imatinib & $\begin{array}{l}\text { Chronic } \\
\text { myeloid } \\
\text { leukemia }\end{array}$ & Novartis & PHARMAC & $\begin{array}{l}\text { Financially- } \\
\text { based }\end{array}$ & Offered overall price reduction & Raftery 2008 [23] \\
\hline & Atorvastatin & Hypertension & Pfizer & PHARMAC & $\begin{array}{l}\text { Financially- } \\
\text { based }\end{array}$ & $\begin{array}{l}\text { Price volume agreements; manufacturer committed to pay for the drug if the sales } \\
\text { exceed a fixed threshold }\end{array}$ & $\begin{array}{l}\text { Antonanzas et al. } \\
2011[24]\end{array}$ \\
\hline & Adalimumab & Arthritis & $\begin{array}{l}\text { Abbott } \\
\text { Laboratories } \\
\text { NZ Ltd }\end{array}$ & PHARMAC & $\begin{array}{l}\text { Financially- } \\
\text { based }\end{array}$ & $\begin{array}{l}\text { Adalimumab spending is probably overstated due to a risk sharing agreement } \\
\text { between the sponsor and PHARMAC, which involves rebates paid by the sponsor } \\
\text { once Government spending reaches a certain level }\end{array}$ & $\begin{array}{l}\text { Access Economics } \\
\text { report for Arthritis New } \\
\text { Zealand } 2010 \text { [25] }\end{array}$ \\
\hline
\end{tabular}

HIRA = Health Insurance Review \& Assessment Service; PLS = positive list system; PHARMAC = The Pharmaceutical Management Agency 


\section{Financially-based schemes}

Financially-based schemes involving price reductions, price-volume agreements, or utilization caps were the most common risk-sharing schemes across the Asia-Pacific region $(77.4 \% ; \mathrm{n}=82)$. We identified 3 in South Korea, 5 in New Zealand, and 74 schemes in Australia. Forty-one of 74 schemes in Australia were hybrid schemes that involved both pricing arrangements and conditional treatment continuation.

\section{Discussion}

In attempts to provide access to promising technologies, many countries are assessing the potential applicability of patient access schemes for their markets; these schemes typically involve novel arrangements between payers and manufacturers. We reviewed the literature on patient access schemes in the last two decades, with a focus on experiences in the Asia-Pacific region. We found a few schemes from South Korean and New Zealand, and many more schemes in Australia. Though different in their development and implementation, these schemes have arisen in response to cost pressures, demands from key stakeholders, and the inherent uncertainties in the health value and estimated budget impact of medical products in real-world settings. Pharmaceuticals account for nearly all identified schemes. The preponderance of pharmaceutical schemes may reflect the different evidence profiles at product launch, the higher budget impact of pharmaceuticals versus devices, and/or the different intellectual property and patent environments. There do not appear to be guidelines on when patient access schemes are applied or which type of scheme is most appropriate in a given circumstance. However, our findings suggest that the main targets are pharmaceuticals likely to have high budget impact due to high per-patient acquisition costs and/or potential large volumes of use, and pharmaceuticals likely to be used beyond their approved indications.

In the Asia-Pacific region, Australia has the most experience with patient access schemes. This may reflect the negotiating power and extensive experience in technology assessment, due to the design of the regulatory, institutional, and policy structures for medicines benefit decisions in the Australian publicly-funded national healthcare system $[4,6]$. High-cost medicines for treatment of cancer and inflammatory diseases were the targets of more than half of the schemes in Australia. Typical outcome-based schemes, in which the price or level of reimbursement is tied to achieving intermediate or final clinical endpoints, have rarely been used in Australia, with bosentan being the only example. Implementing outcome-based schemes is complex: they require detailed longitudinal information on patient clinical status (e.g., disease severity and progression, comorbidities) [30]; they require substantial financial, human, and infrastructure resources for monitoring of patients and financial transactions related to treatments [31]; and they require a mechanism for adjusting price or level of reimbursement when explicit clinical endpoints are not reached. Instead, Australia limits continued subsidy of high-cost medicines to patients who demonstrate an adequate response, known as "conditional treatment continuation" policies, which are often coupled with pricing arrangements, that is, hybrid schemes. Initial access is also restricted to a small pool of patients largely based on disease severity and non-responsiveness to less expensive therapeutic alternatives. Criteria restricting both initial and continued access aim to maximize value-formoney and control costs. While some evidence suggests that such policies can cap spending [28,32], whether they achieve value-for-money or if patients who need the medicines actually gained access should be investigated. Further, strict initial and continued access criteria are ethically challenging as individual patients may just missed the arbitrary threshold for access [33,34].

Evidence generation schemes were also rarely used in the Asia-Pacific region, possibly due to the complexity in tracking patient outcomes and the additional costs and administrative burden involved to operate these schemes.

In contrast, we found that financially-based schemes were common, possibly due to fewer operational challenges. Price-volume agreements and utilization caps have been in place for more than a decade in Australia [19]. Typically, product prices are reduced if sales exceed pre-agreed volumes, or expenditures refunded by the manufacturer if government expenditures exceed a pre-agreed cap or threshold. Price-volume agreements can shift cost considerations from the payer to the manufacturer, which is important especially if there are concerns that (i) new medicines will be prescribed to a wider population than envisaged, (ii) the patients prescribed the drug will not always be those most likely to gain the greatest benefit $[4,11,35]$, and/or (iii) the product does not result in the expected clinical benefits. Expectations are that manufacturers will target promotion in accordance with approved prescribing requirements. Details of patient access schemes (e. g., capped prices, negotiated volumes) are generally unavailable to the public [19].

Our study has several limitations. The review focused on the Asia-Pacific region; however, the investigators were limited to the English language literature; schemes that were described only in other languages have not been included. Given the sensitive nature of contracts in this field, it is likely information on schemes remains unpublished. Manufacturers will be reluctant to disclose details about patient access schemes if such information will be available to other countries that use external 
reference pricing. We thus are likely to have underestimated the total number of patient access schemes in Asia-Pacific countries. Nevertheless, this study provides insights about what types of products are commonly targets for patient access schemes and about recent experiences in the Asia-Pacific region that may inform the development of future schemes.

Although beyond the scope of this review, we noted that industry-sponsored patient assistance programs were implemented in several countries to improve access to medicines. For instance, the Glivec International Patient Assistance Program, implemented since 2001 in 81 low and middle-income countries (including China, India, Indonesia, Thailand, and Vietnam), has provided access to imatinib for patients with specific types of leukemia or gastrointestinal cancer [36]. The MUSANDA patient assistance program, implemented in the United Arab Emirates, supports access to ranibizumab for agerelated macular degeneration. Although payers are usually not involved in industry-sponsored patient assistance programs, these programs can provide interim access to innovative medicines while payer-industry patient access schemes are under negotiation; they can complement payer-industry patient access schemes by providing treatments for individual patients who are unable to self-fund treatments and who have no third-party payer; or they can become a vehicle for implementing a payer-industry patient access scheme, as has happened for coverage of imatinib in Thailand [37].

Globally, forecasts estimate that spending on new biologic agents will outpace overall spending growth on medicines and represent about $20 \%$ of the estimated US $\$ 1.2$ trillion total pharmaceutical market by 2017 [38]. The recent availability of sofosbuvir, a new, curative treatment for hepatitis $\mathrm{C}$, a disease that is highly prevalent in many low and middle-income countries, highlights the need for innovation in medicines financing. Refined patient access schemes may constitute muchneeded innovations in financing arrangements that provide equitable and affordable access for those who need the new medicines. Payers have an incentive to develop strategies that can help control costs while ensuring patient access to medical products that benefit health. Patient access schemes may also mitigate the negative impacts of uncertainties in cost-effectiveness and budget impact estimates, and shift payer and manufacturer focus to improving patient outcomes in real-world settings - the ultimate goal of medical care. Payers have different ranges of authority over pricing, access, and evidence generation; some are more limited in the types of coverage decisions or access schemes they can consider, which may explain some of the variation in use of schemes between countries. Manufacturers are likely to prefer patient access schemes over a denial of coverage or explicitly reduced pricing, in part because patient access schemes can keep the real reimbursement prices confidential allowing them to tier prices by markets without the threat of external reference pricing $[5,13]$.

There is little evidence on whether patient access schemes achieve their intended goals; they are a relatively recent development and the details of such agreements are usually confidential. However, key stakeholders appear willing to at least discuss types of schemes that can enable patient access to needed medicines $[4,31,33]$. Future studies should generate evidence on whether patients who need the medicines actually receive and benefit from them; whether schemes make high quality care more affordable for households and systems; whether they provide incentives for manufacturers to continue to invest in products that meet unmet needs; and whether data collected as part of the schemes confirm estimated cost-effectiveness or long-term benefits. Such evidence about potential benefits and costs is needed to inform the adoption of patient access schemes globally. Other factors that may affect their adoption center around operational challenges such as administrative burden and difficulty in tracking patient outcomes in healthcare delivery and financing systems $[4,5,13,28,30-34,39]$, as well as the transparency and perceived fairness of the complex decisions on the establishment of patient access schemes and criteria for access $[33,34]$. Research is needed to compare costs of administration and operation on the payer side and effects on pricing strategies globally on the pharmaceutical company side between patient access schemes and upfront discounts in drug prices.

\section{Conclusions}

Patient access schemes offer an important option for healthcare systems to allow patient access to promising technologies that may not otherwise be funded. Our study adds to existing knowledge by identifying and characterizing published patient access schemes in the Asia-Pacific region. Financially-based access schemes are most common. Australia tends to couple conditional treatment continuation with financial arrangements to provide further assurance. The main targets of patient access schemes are pharmaceuticals likely to have high budget impact due to high perpatient costs and/or large volumes of use, and pharmaceuticals that may be adopted more widely than indicated. With the rapid proliferation of high-cost medicines, these schemes may increasingly be used to enable access to innovative care within finite budgets. Future research is needed to generate evidence about the effectiveness and economic, administrative, and company pricing policy consequences of patient access schemes. 


\section{Author details}

'Department of Population Medicine, Harvard Medical School and Harvard Pilgrim Health Care Institute, Boston, USA. ${ }^{2}$ Jefferson Medical School, Philadelphia, USA. ${ }^{3}$ School of Pharmacy, University of Auckland, Auckland, New Zealand.

Received: 8 September 2014 Accepted: 4 December 2014 Published online: 16 February 2015

\section{References}

1. Tangcharoensathien V, Patcharanarumol W, Ir P, Aljunid S, Mukti A, Akkhavong $K$, et al. Health-financing reforms in Southeast Asia: challenges in achieving universal coverage. Lancet. 2011;377(9768):863-73.

2. World Health Organization: What is Universal Health Coverage? http://www.who.int/features/qa/universal_health_coverage/en/. Accessed February 23, 2015.

3. Freemantle N, Hill S. Evaluating Pharmaceuticals for Health Policy and Reimbursement. Oxford, UK: Blackwell; 2004

4. Lu CY, Williams K, Day R, March L, Sansom L, Bertouch J. Access to high cost drugs in Australia. BMJ. 2004;329(7463):415-6.

5. Walker S, Sculpher M, Claxton K, Palmer S. Coverage with evidence development, only in research, risk sharing, or patient access scheme? A framework for coverage decisions. Value Health. 2012;15(3):570-9.

6. Sansom L. The subsidy of pharmaceuticals in Australia: processes and challenges. Aust Health Rev. 2004;28(2):194-205.

7. Jirawattanapisal T, Kingkaew P, Lee TJ, Yang MC. Evidence-based decisionmaking in Asia-Pacific with rapidly changing health-care systems: Thailand South Korea, and Taiwan. Value Health. 2009;12 Suppl 3:S4-S11.

8. Thatte U, Hussain S, de Rosas-Valera M, Malik MA. Evidence-based decision on medical technologies in Asia Pacific: experiences from India, Malaysia, Philippines, and Pakistan. Value Health. 2009;12 Suppl 3:S18-25.

9. Teerawattananon Y, Tantivess S, Yothasamut J, Kingkaew P, Chaisiri K. Historical development of health technology assessment in Thailand. Int J Technol Assess Health Care. 2009;25 Suppl 1:241-52.

10. PHARMAC: Prescription for Pharmacoeconomic Analysis: Methods for costutility analysis. 2012; http://www.pharmac.health.nz/assets/pfpa-final.pdf. Accessed February 23, 2015.

11. Towse A, Garrison Jr LP. Can't get no satisfaction? Will pay for performance help?: toward an economic framework for understanding performancebased risk-sharing agreements for innovative medical products. Pharmacoeconomics. 2010;28(2):93-102.

12. Lu CY. Uncertainties in real-world decisions on medical technologies. Int J Clin Pract. 2014;68(8):936-40.

13. Carlson JJ, Sullivan SD, Garrison LP, Neumann PJ, Veenstra DL. Linking payment to health outcomes: a taxonomy and examination of performance-based reimbursement schemes between healthcare payers and manufacturers. Health Policy. 2010;96(3):179-90.

14. Australian Government Department of Human Services: Complex Authority Required Highly Specialised Drugs (CAR HSD). http://www.humanservices.gov. au/health-professionals/services/highly-specialised-drugs/. Accessed February 23, 2015

15. Stafinski T, McCabe CJ, Menon D. Funding the unfundable: mechanisms for managing uncertainty in decisions on the introduction of new and innovative technologies into healthcare systems. Pharmacoeconomics. 2010;28(2):113-42.

16. Australian Government Department of Health: Pharmaceutical Benefits Scheme Schedule. http://www.pbs.gov.au/pbs/home. Accessed February 23, 2015.

17. Australian Government Department of Health: Therapeutic Relativity Sheets. http://pbs.gov.au/info/industry/pricing/pbs-items/therapeutic-relativitysheets. Accessed February 23, 2015

18. Australian Government Department of Health: Public Summary Documents. http://www.pbs.gov.au/pbs/industry/listing/elements/pbac-meetings/psd. Accessed February 23, 2015.

19. Robertson J, Walkom EJ, Henry DA. Transparency in pricing arrangements for medicines listed on the Australian Pharmaceutical Benefits Scheme. Aust Health Rev. 2009;33(2):192-9.

20. Adamski J, Godman B, Ofierska-Sujkowska G, Osińska B, Herholz H, Wendykowska K, et al. Risk sharing arrangements for pharmaceuticals: potential considerations and recommendations for European payers. BMC Health Serv Res. 2010;10:153.
21. Pugatch M, Healy P, Chu R: Sharing the Burden: Could Risk-Sharing Change the Way We Pay for Healthcare? The Stockholm Network; 2010. http://www stockholm-network.org/downloads/publications/Sharing_the_Burden.pdf. Accessed February 23, 2015.

22. Lee EK, Kim BY, Lim JY, Park MH. Different policy outcomes of the new drugs and currently listed drugs under the positive list system in South Korea. Value Health. 2012;15(1 Suppl):S100-3.

23. Raftery JP. Paying for costly pharmaceuticals: regulation of new drugs in Australia, England and New Zealand. Med J Aust. 2008;188(1):26-8.

24. Antonanzas F, Juarez-Castello C, Rodriguez-lbeas R. Should health authorities offer risk-sharing contracts to pharmaceutical firms? A theoretical approach. Health Econ Policy Law. 2011;6(3):391-403.

25. Access Economics Pty Limited. The Economic Cost of Arthritis in New Zealand in 2010: Arthritis New Zealand; 2010. http://www.arthritis.org.nz/wp-content/ uploads/2011/07/economic-cost-of-arthritis-in-new-zealand-final-print.pdf. Accessed February 23, 2015.

26. PHARMAC: Decision Criteria. http://www.pharmac.health.nz/medicines/ how-medicines-are-funded/decision-criteria/. Accessed February 23, 2015

27. Yang BM, Bae EY, Kim J. Economic evaluation and pharmaceutical reimbursement reform in South Korea's National Health Insurance. Health Aff. 2008;27(1):179-87.

28. Lu CY, Williams KM, Day RO. Access to tumour necrosis factor inhibitors for rheumatoid arthritis treatment under the Australian Pharmaceutical Benefits Scheme: are we on target? Intern Med J. 2006;36(1):19-27.

29. Owen A, Spinks J, Meehan A, Robb T, Hardy M, Kwasha D, et al. A new model to evaluate the long-term cost effectiveness of orphan and highly specialised drugs following listing on the Australian Pharmaceutical Benefits Scheme: the Bosentan Patient Registry. J Med Econ. 2008;11(2):235-43.

30. Lu CY, Williams KM, Day RO. Accessing health outcome data on high-cost medicines in Australia. Med J Aust. 2006;184(8):411-3.

31. Lu CY, Ritchie J, Williams K, Day R. The views of stakeholders on controlled access schemes for high-cost antirheumatic biological medicines in Australia. Austr N Z Health Policy. 2007:4:26.

32. Lu CY, Williams KM, Day RO. The funding and use of high-cost medicines in Australia: the example of anti-rheumatic biological medicines. Austr N Z Health Policy. 2007:4:2.

33. Lu CY, Macneill P. Williams K, Day R. Access to high cost medicines in Australia: ethical perspectives. Austr N Z Health Policy. 2008;5:4.

34. Lu CY, Williams KM, March L, Bertouch JV, Day RO. Subsidised access to TNF alpha inhibitors: is the rationale for exclusion of rheumatoid-factor-negative patients defensible? Med J Aust. 2004;181(8):457. discussion 457-458.

35. McCabe CJ, Stafinski T, Edlin R, Menon D, Banff AEDS. Access with evidence development schemes: a framework for description and evaluation. Pharmacoeconomics. 2010:28(2):143-52.

36. Lassarat S, Jootar S. Ongoing challenges of a global international patient assistance program. Ann Oncol. 2006:17 Suppl 8:viii43-6.

37. Ministry of Public Health. Notification of the Ministry of Public Health: Re: Exercising of right on pharmaceuticals products patent for imatinib, dated 25th January, B.E. 2551. Nonthaburi: Ministry of Public Health; 2008.

38. IMS Institute for Healthcare Informatics: The Global Use of Medicines: Outlook through 2017. 2013; http://www.imshealth.com/deployedfiles/imshealth/ Global/Content/Corporate/IMS\%20Health\%2OInstitute/Reports/Global_ Use of Meds_Outlook_2017/IHH_Global_Use_of_Meds_Report_2013.pdf. Accessed February 23, 2015.

39. Lu CY, Williams K Day R. Access to biologic medicines for the treatment of rheumatic diseases: lessons from Australia. Int J Rheum Dis. 2008;11:11-4 